\title{
Entropy potential and Lyapunov exponents
}

\author{
Stefano Lepri ${ }^{1 \dagger}$, Antonio Politi ${ }^{2 \ddagger}$ and Alessandro Torcini ${ }^{3 \dagger}$ \\ 1 Max-Planck-Institut für Physik Komplexer Systeme D-01187 Dresden, Germany \\ ${ }^{2}$ Istituto Nazionale di Ottica I-50125 Firenze, Italy \\ 3 Dipartimento di Energetica "S. Stecco" I-50139 Firenze, Italy
}

(September 18, 2018)

\begin{abstract}
According to a previous conjecture, spatial and temporal Lyapunov exponents of chaotic extended systems can be obtained from derivatives of a suitable function: the entropy potential. The validity and the consequences of this hypothesis are explored in detail. The numerical investigation of a continuous-time model provides a further confirmation to the existence of the entropy potential. Furthermore, it is shown that the knowledge of the entropy potential allows determining also Lyapunov spectra in general reference frames where the time-like and space-like axes point along generic directions in the space-time plane. Finally, the existence of an entropy potential implies that the integrated density of positive exponents (Kolmogorov-Sinai entropy) is independent of the chosen reference frame.
\end{abstract}

PACS numbers: 05.45.+b

$\dagger$ also Istituto Nazionale di Fisica della Materia I-50125 Firenze, Italy $\ddagger$ also Istituto Nazionale di Fisica Nucleare, Sezione di Firenze I-50125 Firenze, Italy 


\section{Lead paragraph}

Out-of-equilibrium systems give rise to a rich variety of phenomena which are often dealt with ad hoc methods. Perhaps the main difficulty lying behind the development of general tools is the lack of an Ansatz equivalent to the Gibbs-Boltzmann weight, which allows estimating a priori the probability of each configuration of an equilibrium system. Although the problem cannot be (easily) overcome, it is tempting to explore the possibility to extend and apply concepts like Lyapunov exponents to extract information about the invariant measure of a high-dimensional system. For instance, on the one hand it is well known that, through the Kaplan-Yorke formula, it is possible to estimate the fractal dimension of a finite (low) dimensional attractor; on the other hand, however, it is unclear how the local dynamics arising in different regions of an extended system combine together to determine the global invariant measure. It is our opinion that a sensible answer will be given only after having extended the Lyapunov analysis, to account for spatial propagation as well as temporal instabilities. The chronotopic approach, introduced in Ref. [1,2] represents a tentative construction that goes in this direction, introducing a sort of dispersion relations in chaotic systems. In this paper, we test the general validity of the method both with numerical simulations and by investigating its internal coherence. 


\section{INTRODUCTION}

Lyapunov exponents, providing information on the evolution in tangent space are very useful in that they allow characterizing the invariant measure [3]. This is particularly clear in the case of hyperbolic systems as revealed by the construction of the Bowen-Ruelle-Sinai measure [4], but is also effectively true for the general and more realistic class of smooth nonhyperbolic systems. However, an effective exploitation of these ideas in spatially extended systems is hindered by the infinite-dimensionality of the phase-space and by the existence of propagation phenomena that requires a more detailed understanding besides that one provided by the knowledge of the usual Lyapunov spectrum [5]. In order to reach a more complete comprehension of phenomena occurring in tangent space, two families of Lyapunov spectra characterizing temporal, resp. spatial, dynamics of infinitesimal perturbations have been introduced and discussed [1]. Subsequently [2], it has been shown that the two families are not independent of one another. More precisely, it has been conjectured that all the information on Lyapunov exponents can be obtained from a single observable: the entropy potential $\Phi$, a function of the temporal and the spatial growth rates $\lambda, \mu$, respectively. The name "entropy potential" follows from the observation that $\Phi(\lambda=0, \mu=0)$ coincides with the density of Kolmogorov-Sinai entropy [6]. However, such a conjecture is based only on a few simple examples that can be analytically worked out and on numerical simulations performed for a chain of coupled maps. One of the aims of the present paper is to strengthen the validity of the conjecture by performing new tests in a more realistic system.

Moreover, we intend to show a further connection between spatial and temporal Lyapunov exponents by studying the evolution of perturbations along generic "world-lines" in the space-time, i.e. along directions other than the natural space and time axes. The extension of the usual definition of Lyapunov exponents to this more general class of frames, partially discussed in [7], is rather appropriate for characterizing patterns with some anisotropy. Here, we prove that this seemingly more general class of spatiotemporal exponents can be derived from the knowledge of spatial and temporal Lyapunov spectra, which thus confirm to contain all the relevant information.

The present paper is organized as follows. In Section II we recall the definition of the entropy potential and present some new test for its existence. Sec. III is devoted to Lyapunov analysis in tilted reference frames i.e. to the definition of spatiotemporal exponents, while Sec. IV deals with their relationships with more standard dynamical indicators. Some conclusive remarks are finally reported in Sec. V.

\section{LYAPUNOV SPECTRA FROM AN ENTROPY POTENTIAL}

In this Section, we first introduce the main classes of models employed in the investigation of spatio-temporal chaos. Then, we recall the notion of temporal (TLS) and spatial (SLS) Lyapunov spectra with a particular emphasis to their connection with the entropy potential. The very existence of the latter is then investigated in the last part, for a specific continuoustime model.

Reaction-diffusion systems are among the most relevant models for the study of spatiotemporal chaos. The standard form for the evolution equations is [5] 


$$
\partial_{t} \mathbf{u}=\mathbf{F}(\mathbf{u})+\mathbf{D} \partial_{x}^{2} \mathbf{u}
$$

where the field $\mathbf{u}(x, t)$ is defined on the domain $[0, L]$, with periodic boundary conditions $\mathbf{u}(0, t)=\mathbf{u}(L, t)$. The nonlinear function $\mathbf{F}$ accounts for the local reaction dynamics, while the matrix $\mathbf{D}$ represents the spatial coupling induced by diffusion.

A simplified class of models can be obtained by spatial discretization, i.e. by considering a 1D lattice of coupled oscillators [8]

$$
\dot{\mathbf{u}}_{i}=\mathbf{F}\left(\mathbf{u}_{i}\right)+D\left(\mathbf{u}_{i+1}-2 \mathbf{u}_{i}+\mathbf{u}_{i-1}\right)
$$

where the index $i$ labels each site of a lattice of length $L$ (assuming again periodic boundary conditions).

A further simplification is achieved by discretizing also the time, i.e by considering coupled map lattices (CML) [9], that in their usual form read as

$$
u_{n+1}^{i}=f\left(\frac{\varepsilon}{2} u_{n}^{i+1}+(1-\varepsilon) u_{n}^{i}+\frac{\varepsilon}{2} u_{n}^{i-1}\right),
$$

where $n$ is the time index, and $\varepsilon$ gauges the diffusion strength. The function $f$, mapping a given interval $I$ of the real axis onto itself, simulates the nonlinear reaction process. In particular, we will focus on the homogeneous $f(x)=r x(\bmod 1)$ and logistic $f(x)=$ $4 x(1-x)$ CML with $u_{n}^{i} \in[0,1]$.

CML models have been also proposed to mimic 1D open-flow systems [10], characterized by flux terms in the field equation. For example the model

$$
u_{n+1}^{i}=f\left(\varepsilon(1-\alpha) u_{n}^{i-1}+(1-\varepsilon) u_{n}^{i}+\varepsilon \alpha u_{n}^{i+1}\right)
$$

with the parameter $\alpha$ bounded between 0 and 1, accounts for the possibility of an asymmetric coupling, corresponding to first order derivatives in the continuum limit.

Let us denote with $\delta(x, t)$ a generic perturbation that can be assumed to possess an exponential profile both in space and time,

$$
\delta(x, t)=a(x, t) \exp (-\mu x+\lambda t)
$$

Depending whether $\mu$, or $\lambda$ is considered as a free parameter, one can define either the temporal or the spatial Lyapunov spectrum. Let us first discuss the temporal Lyapunov spectrum $n_{\lambda}(\lambda, \mu)$ : it is obtained in the usual way by following the evolution in tangent space of a perturbation of the form $b(x, t) e^{\mu x}$, where $\mu$ is fixed a priori. Obviously, for $\mu=0$, one recovers the standard Lyapunov spectrum. Alternatively, one can consider the temporal growth rate $\lambda$ as a free parameter and thereby determine the spatial spectrum $n_{\mu}(\lambda, \mu)$. The latter procedure is symmetric to the previous one: $\mu$ and $\lambda$ are mutually exchanged, as well as $x$ and $t$, i.e. the tangent dynamics is followed along the spatial direction.

As a result of the above two approaches one is confronted with a set of four variables: $\mu$, $\lambda, n_{\mu}$, and $n_{\lambda}$. The main conclusion of [1] is that any two of them can be taken as independent variables, the other two variables providing a complete characterization of spatio-temporal stability properties. Furthermore, in [2], we have conjectured that both integrated densities are not actually independent of one another, but can be obtained from a single function $\Phi(\lambda, \mu)$, 


$$
\begin{aligned}
& \partial_{\lambda} \Phi=n_{\lambda} \\
& \partial_{\mu} \Phi=n_{\mu} \quad,
\end{aligned}
$$

that is called entropy potential as it coincides with the usual Kolmogorov-Sinai entropy density along a suitable line (see Sec. IV).

Since equivalent descriptions are obtained by choosing any pair of independent variables, the appropriate entropy potential is obtained by means of a Legendre transform in the new variables. For instance, if one wishes to consider $\lambda$ and $n_{\lambda}$ as new conjugate variables, the new entropy potential $\Psi\left(n_{\lambda}, \lambda\right)$ is given by

$$
\Psi \equiv \lambda n_{\lambda}-\Phi
$$

and the following relations hold

$$
\begin{aligned}
& \frac{\partial \Psi}{\partial n_{\lambda}}=\lambda \\
& \frac{\partial \Psi}{\partial \mu}=-n_{\mu} .
\end{aligned}
$$

The existence of a potential has been conjectured on the basis of analyticity properties of the relationship linking $\mu$ and $\lambda$. The key steps to prove, e.g. (8), are (i) the introduction of complex spatial and temporal "growth rates" $\tilde{\mu}$ and $\tilde{\lambda}$, respectively; (ii) the identification (apart from a sign and a multiplicative factor) of $\operatorname{Im}(\tilde{\lambda})$ and $\operatorname{Im}(\tilde{\mu})$ with $n_{\mu}$ and $n_{\lambda}$, respectively (obviously, $\operatorname{Re}(\tilde{\lambda})=\lambda$ and $\operatorname{Re}(\tilde{\mu})=\mu$ ).

As a matter of fact, we have been able to substantiate the second point only in simple cases where the linear stability analysis reduces to the an eigenvalue equation for a fixed point or a periodic orbit. In the fully aperiodic regime, there is only numerical evidence in a chain of coupled logistic maps, where a test on the existence of a potential has been performed by computing a certain circulation integral [2].

In order to test the general validity of our main Ansatz, we consider now a continuoustime model. Since the generation mechanisms of the multipliers in tangent space are not important for our conclusions, we have assumed that they are the result of stochastic processes, thus escaping the need to integrate also some nonlinear equations in phase-space. We shall refer to a model of coupled oscillators (see Eq. 2), whose evolution in tangent space can be written as

$$
\dot{\delta u_{i}}=m_{i}(t) \delta u_{i}+D\left(\delta u_{i+1}+\delta u_{i-1}\right)
$$

where the random multipliers $m_{i}(t)$ are indirectly defined through their power spectrum

$$
S(\omega)=S_{0}\left[\exp \left(-\frac{\left(\omega-\omega_{0}\right)^{2}}{\sigma}\right)+\exp \left(-\frac{\left(\omega+\omega_{0}\right)^{2}}{\sigma}\right)\right] .
$$

This choice implies that $m_{i}(t)$ is an analytic function of time and possesses some degree of periodicity as one expects to be the case of many deterministic chaotic signals.

From a numerical point of view, we have decided to compute the derivative appearing in (9) through a simple finite difference (Euler) scheme. This approach, although not the most 
refined one, allows treating in a consistent way the temporal integration and the recursive iteration in space. Indeed, by expressing the perturbation as $\delta u_{i}(t)=v_{i}(t) \exp (-\mu i+\lambda t)$, Eq. (9) can be rewritten as

$$
v_{i}(t+\Delta t) \mathrm{e}^{\lambda \Delta t}=v_{i}(t)+\Delta t\left[m_{i}(t) v_{i}+D\left(v_{i+1} \mathrm{e}^{\mu}+v_{i-1} \mathrm{e}^{-\mu}\right)\right],
$$

which can be easily iterated either in space or time to determine the TSL and the SLS. In principle, one should choose a sufficiently small integration time step; in practice the spatial recursion becomes soon unfeasible, since the dimension of the "temporal" phase space is proportional to $1 / \Delta t$. Accordingly, we have considered a few different values of $\Delta t$, namely $0.2,0.1$, and 0.05 , paying more attention to testing the existence of the potential in each case, rather than to computing the actual limit spectra (i.e. to the limit $\Delta t \rightarrow 0$ ). In fact, although for a finite value of $\Delta t$, one cannot assert, rigorously speaking, to have simulated Eq. (2), model (11) can nevertheless be considered as a dynamical system in itself worth being studied (actually, it is a CML with a different coupling scheme from that usually assumed).

In Fig. 1, we have reported the borders of the spectra in the $(\mu, \lambda)$-plane as estimated from the SLS and the TLS. The agreement between the two sets of lines for every value of $\Delta t$ provides a first indication that each approximation of the continuous model is in itself consistent with the findings of [1]. Each border is made of 4 distinct lines: an upper and a lower curve representing the set of maxima and minima of TLS; two symmetric lines representing the extrema of the SLS. For $\Delta t \rightarrow 0$, we expect that the first two curves converge to an asymptotic shape, while the latter two diverge to $\pm \infty$. In fact, the maximum and the minimum spatial divergence rate are limited by the time step $\Delta t$. This is a general feature of continuous time systems as already remarked in [1].

The variation of the lateral borders with $\Delta t$ indeed confirms our expectations, while the dependence of the upper and lower curves (especially at high $|\mu|$-values) indicates, instead, that the convergence to the asymptotic form is rather slow: this is clearly confirmed by the comparison with the border of the TLS determined for a much smaller value of $\Delta t(0.00625)$ (see solid curve in Fig. 1).

In any case, the most relevant test that we wanted to perform concerns the the existence of an entropy potential $\Phi$. This can be numerically done by computing the integral

$$
\int_{A}^{B}\left(n_{\mu}, n_{\lambda}\right) \cdot \vec{w} d s
$$

along different paths joining generic pairs $A, B$ of points in the $(\mu, \lambda)$ plane. If the integral is independent of the path, we have a clear indication of the existence of a potential. All tests that we have performed for different choices of points and of the time-integration steps have been successfull, yielding results that are equal within the numerical accuracy. In Table I, we report a subset of the results, all referring to the same end points but different values of $\Delta t$. We consider this as a further clear evidence that the potential entropy indeed exists and is a rather general feature of at least $1 \mathrm{D}$ spatio-temporal systems.

\section{SPATIOTEMPORAL EXPONENTS}

In the perspective of a complete characterization of space-time chaos, one should consider the possibility of viewing a generic pattern as being generated along directions other than 
time and space axes. In fact, once a pattern is given, any direction can, a priori, be considered as an appropriate "time" axis. Accordingly, questions can be addressed about the statistical properties of the pattern when viewed in that way. The extension of the Lyapunov formalism to generic orientations of the space-time coordinates does not follow simply from an abstract need of completeness; it also results from the attempt of generalizing the nonlinear timeseries analysis to patterns. The existence of low-dimensional chaos led many researchers to investigate the possibility whether a given irregular temporal signal might be the consequence of a few nonlinearly coupled degrees of freeedom. The existence of space-time chaos leads to the equivalent question whether a given pattern is the result of a deterministic 1D nonlinear process. At variance with temporal signals which can be generated only by moving forward or backward in time, in the case of patterns, the identification of the most appropriate spatial and temporal directions is a new and unavoidable element of the game. For this reason, in the next subsection we shall introduce the notion of spatiotemporal exponents.

\section{A. Definitions}

For the moment, we assume that the pattern is continuous both along space and time directions; we shall discuss later how the definitions can be extended to CML models. Let us consider a given spatiotemporal configuration of the field $\mathbf{u}(x, t)$, generated, say, by integrating Eq. (11). When arbitrary directions are considered in the $(x, t)$ plane, the coordinates must be properly scaled in order to force them to have the same dimension. We choose to multiply the time variable by $c$, where $c$ is a suitable constant with the dimension of a velocity. Moreover, let $\vartheta$ denote the rotation angle of the tilted frame $\left(x^{\prime}, c t^{\prime}\right)$ with respect to the initial one $(x, c t)$, adopting the convention that positive angles correspond to clockwise rotations. Sometimes it will be more convenient to identify the new frame by referring to the velocity $v=c \tan \vartheta$. The limit cases $v=0(\vartheta=0)$ and $v=+\infty(\vartheta=\pi / 2)$ correspond to purely temporal and purely spatial propagations, respectively. The coordinate transformation reads as

$$
\begin{aligned}
& c t^{\prime}=\beta\left(c t+\frac{v}{c} x\right) \\
& x^{\prime}=\beta(-v t+x),
\end{aligned}
$$

where $\beta \equiv 1 / \sqrt{\left(1+v^{2} / c^{2}\right)}$. The physical meaning of $v$ is transparent: it can be interpreted as the velocity in the old frame of a point stationary in the tilted frame (constant $x^{\prime}$ ).

The new field $\mathbf{u}\left(x^{\prime}, c t^{\prime}\right)$ can be thought of as being the result of the integration of the model derived from the original one after the change of variables (13). Although it is not obvious whether the invariant measure in the initial frame is still attracting in the new frame (see Ref. [7] for a discussion of this point), one can anyhow study the stability properties by linearizing and defining the Lyapunov exponents in the usual way.

In CML models, the discreteness of both the space and the time lattice leads to some difficulties in the practical construction of tilted frames. In fact, only rational values of the velocity $v$ can be realized in finite lattices (in this case, it is natural to assume that the lattice spacing is the "same" along the spatial and the temporal directions and, accordingly, to set $c=1$ ). Moreover, writing the explicit expression of the model requires introducing 
different site types. For this reason, we discuss in the following the simplest nontrivial case $v=1 / 2$, the generalization to other rational velocities being conceptually straightforward.

A generic spatial configuration in the tilted frame is defined by sites of the spatiotemporal lattice $(i, n)$ connected by alternating horizontal (as in the usual case) and diagonal bonds (see Fig. 2). By suitably adjusting the relative fraction of the two types of links, all rotations between 0 and $\pi / 4$ can be reproduced. The explicit expression of the updating rule requires a proper numbering of the consecutive sites. Moreover, as seen in Fig. 2, it involves the "memory" of two previous states.

Finally, an exact implementation of the mapping rule requires acausal boundary conditions, since the knowledge of future (in the original frame) states is required [7] (this is a general problem occurring also in the continuous case). As we are interested in the thermodynamic limit, we bypass the problem by choosing periodic boundary conditions. Such a choice has been shown not to affect the bulk properties of the dynamical evolution [7].

In the updating procedure, two different cases are recognized: the variable $u$ is either determined from the past values in the neighbouring sites, or it requires the newly updated $u$-value on the right neighbour (see Fig. 2). For $v=1 / 2$, this can be done by simply distinguishing between even and odd sites,

$$
\begin{aligned}
& X_{n+1}^{2 i}=f\left(\frac{\varepsilon}{2} Y_{n}^{2 i-1}+(1-\varepsilon) X_{n}^{2 i}+\frac{\varepsilon}{2} X_{n}^{2 i+1}\right) \\
& X_{n+1}^{2 i+1}=f\left(\frac{\varepsilon}{2} X_{n}^{2 i}+(1-\varepsilon) X_{n}^{2 i+1}+\frac{\varepsilon}{2} X_{n+1}^{2 i+2}\right)
\end{aligned}
$$

where $i=1, \ldots, L / 2$ ( $L$ is assumed to be even for simplicity), while

$$
Y_{n+1}^{j} \equiv X_{n}^{j}
$$

are additional variables introduced to account for the dependence at time $n-1$. Taking into account that $X_{n+1}^{2 i+2}$ can be determined from the $X$ and $Y$ variables at time $n$, the mapping can be finally expressed in the usual synchronous form $\left(X_{n}^{i}, Y_{n}^{i}\right) \rightarrow\left(X_{n+1}^{i}, Y_{n+1}^{i}\right)$, but with an asymmetric spatial coupling with next and next-to-next nearest neighbours. The Lyapunov exponents $\eta_{j}$ can now be computed with the usual technique [11].

In analogy with the original model, we expect again that, in the limit of infinitely extended systems, the set of exponents $\eta_{j}(v)$ will converge to an asymptotic form,

$$
\eta_{j}(v) \rightarrow \eta\left(v, n_{\eta}\right)
$$

where $n_{\eta}=j / L$ is the corresponding integrated density. We will refer to this function as the spatiotemporal Lyapunov spectrum (STLS). In the limit cases $v=0,+\infty(\vartheta=0, \pi / 2)$, the STLS reduces to the standard temporal and spatial spectrum, respectively.

The recursive scheme (14) implies an increase of the phase-space dimension by a factor $(1+1 / 2)$ (in general $1+v)$. Actually, as we will argue, these new degrees of freedom are not physically relevant. However, for consistency reasons with the original rescaling of the spatial variable, we choose to normalize the spatiotemporal density between 0 and $1+v$ (the time units are, instead, left unchanged by the above construction).

The generalization to asymmetric maps (田) is straightforward: it removes the degeneracy $v \rightarrow-v$. Numerical results for logistic maps, indicate that the dependence of the positive 
exponents on the velocity is quite weak in the fully symmetric case $\alpha=1 / 2$ (for instance, the maximum exponent exhibits a $20 \%$ variation in the whole $v$ range), while it is remarkable for asymmetric couplings. In every case, the negative part of the spectrum sharply changes with the velocity. This is consistent with the results obtained for delayed maps in Ref. [7].

\section{B. Representation in the $(\mu, \lambda)$ plane}

Spatiotemporal exponents can be put in relation with $\mu$ and $\lambda$ by rewriting the general expression for a perturbation in a frame rotated by an angle $\vartheta$,

$$
\exp (\mu x+\lambda t)=\exp \left(\mu^{\prime} x^{\prime}+\lambda^{\prime} t^{\prime}\right)
$$

Such an equation induces a rotation of the same angle in the $(c \mu, \lambda)$ variables,

$$
\begin{aligned}
& \lambda^{\prime}=\beta(\lambda+v \mu) \\
& \mu^{\prime}=\beta\left(-\left(v / c^{2}\right) \lambda+\mu\right) .
\end{aligned}
$$

The above equations allow studying the stability with respect to generic perturbations with an exponential profile along $x^{\prime}$. For simplicity, we shall consider only uniform perturbations,

$$
\exp (\mu x+\lambda t)=\exp \left(\eta t^{\prime}\right)
$$

where the growth rate $\eta$ denotes the spatiotemporal exponent. Notice that we have changed notations from $\lambda^{\prime}$ to $\eta$, to understand that the condition $\mu^{\prime}=0$ is fulfilled. From the second of Eq. (18), uniform perturbations in the rotated frame correspond to points along the line $\mathcal{L}$

$$
\lambda=c^{2} \mu / v
$$

in the $(\mu, \lambda)$ plane.

Whenever the evolution of an exponentially localized perturbation of type (17) is considered, it is natural to introduce the quantity $\hat{V}=\lambda / \mu$, which can be interpreted as the velocity of the front [12]. Eq. (20) connects this velocity with that of the rotated frame,

$$
\hat{V}=c^{2} / v
$$

Therefore, on the basis of definition (19), $\left(x^{\prime}, c t^{\prime}\right)$ can be interpreted as the reference frame in which the front associated with the perturbation propagates with an "infinite" velocity.

The explicit expression for $\eta$ is

$$
\eta=\operatorname{sign}(\lambda) \sqrt{\lambda^{2}+(c \mu)^{2}}=\lambda / \beta
$$

Such a relation can be turned into a self-consistent equation for the maximum Lyapunov exponent $\eta_{\max }$ by imposing the constraint that the pair $(\mu, \lambda)$ lies on the upper border $\lambda=\lambda_{\max }(\mu)$ of the spectrum of the Lyapunov exponents, namely

$$
\eta_{\max }=\frac{1}{\beta} \lambda_{\max }\left(\mu=\frac{v}{c^{2}} \beta \eta_{\max }\right)
$$


Some ambiguities arise when velocities $v>c$ are considered, since the line $\mathcal{L}$ intersects $\lambda_{\max }(\mu)$ in two points as seen in Fig. 3. This phenomenon was already noticed in Ref. [12], while discussing the propagation of exponentially localized disturbances in the original reference frame. Moreover, it has been shown that only the front corresponding to the smaller value of $\mu$ is stable, except for some cases where a nonlinear mechanism intervenes dominating the propagation process [13].

At $v=c^{2} / V_{*}$ the two intersections degenerate into a single tangency point. This condition defines $V_{*}$, which can be interpreted as the slowest propagation velocity of initially localized disturbances [12].

The extension of Eq. (23) to the rest of the spectrum requires to connect $n_{\lambda}$ and $n_{\mu}$ with $n_{\eta}$. In the next section, we will show how to perform such a step with the help of the entropy potential. Here, we limit ourselves to discuss the structure of the STLS for different values of the tilting angle $\vartheta$. In Fig. 1 , we report the borders of the bands, which can be determined from the intersections of $\mathcal{L}$ with the border $\partial \mathcal{D}$ of the domain of allowed perturbations (see Fig. 1 of Ref. [1] and Fig. 3). For $\vartheta=0$ (temporal case) a single band is present but, as soon as $\vartheta>0$, a second negative band arises from the intersections with the branch diverging to $-\infty$ at $\mu=-\mu_{c}$. For $\vartheta>\pi / 4$, the negative band disappears and a positive band arises from the intersections with the branch diverging to $+\infty$ with slope $v=c$. A single band spectrum is again recovered for $\vartheta \geq \vartheta_{*}=\operatorname{atan}\left(c / V_{*}\right)$.

Notice that in symplectic maps, the STLS is symmetric for any value of $\vartheta$ (see Ref. [1]) so that positive and negative bands appear and disappear simultaneously.

It is worthwhile to illustrate some of the above considerations in the simple example of the linear diffusion equation

$$
\partial_{t} u=\gamma u+D \partial_{x}^{2} u
$$

that arise when dealing with the linear stability analysis of uniform and stationary solutions $u(x, t)=u^{*}$ of the scalar version of Eq. (11). The expression for $\lambda_{\max }(\mu)$ can be obtained by assuming $u(x, t)=\exp (\mu x+\lambda t)$, so that

$$
\lambda_{\max }(\mu)=\gamma+D \mu^{2}
$$

Accordingly, Eq. (23) reads as

$$
\beta \eta_{\max }=\gamma+D\left(\frac{v}{c^{2}} \beta \eta_{\max }\right)^{2}
$$

On the other hand, the model equation in the rotated frame can be obtained from the substitutions

$$
\begin{aligned}
& \partial_{t} \rightarrow \beta\left(\partial_{t^{\prime}}-v \partial_{x^{\prime}}\right) \\
& \partial_{x} \rightarrow \beta\left(\frac{v}{c^{2}} \partial_{t^{\prime}}+\partial_{x^{\prime}}\right)
\end{aligned}
$$

By introducing the usual Ansatz for the shape of the perturbation,

$$
u\left(x^{\prime}, t^{\prime}\right) \sim \exp \left[i \kappa x^{\prime}+(\eta+i \Omega) t^{\prime}\right]
$$


separating the real from the imaginary part, and eliminating $\Omega$, we obtain the integrated density of spatiotemporal exponents

$$
\kappa(\eta, v)=\beta\left(1-2 D \frac{v^{2}}{c^{4}} \beta \eta\right) \sqrt{\frac{v^{2}}{c^{4}}(\beta \eta)^{2}-\frac{\beta \eta}{D}+\frac{\gamma}{D}}
$$

and the corresponding STLS $\eta(\kappa, v)$. Dimensional analysis shows that $\kappa$ is an inverse length, as expected for a density of exponents. Notice that Eq. (26) is recovered, by setting $\kappa=$ $\Omega=0$ in Eq. (29).

In this and more general continuous models, we should remark that the line $\mathcal{L}$ intersects $\lambda_{\max }(\mu)$ twice for any arbitrarily small $v$. This is because the Laplacian operator sets no upper limit to the propagation velocity of disturbances.

\section{FROM THE ENTROPY POTENTIAL TO DYNAMICAL INVARIANTS}

The present section is devoted to establish the consequences of the existence of the entropy potential on the Lyapunov spectra and other dynamical indicators such as the Kolmogorov-Sinai entropy and the Kaplan-Yorke dimension of the attractor. In order to keep the notations as simple as possible, we assume that time and space coordinates are scaled in such a way that $c=1$.

\section{A. Spatiotemporal exponents}

The very existence of the entropy potential $\Phi$ implies that the Lyapunov spectrum in a frame tilted at an angle $\vartheta$ (recall that $\vartheta$ is the angle from the $\lambda$-axis) can be obtained by computing the derivative of $\Phi$ along the direction $\vec{u}=(\sin \vartheta, \cos \vartheta)$ in the $(\mu, \lambda)$ plane. In fact, this is a straightforward generalization of the previous findings that $n_{\mu}$ and $n_{\lambda}$ are the derivatives of $\Phi$ along the $\mu$ and $\lambda$ direction, respectively. Accordingly, the STLS is linked to the TLS and SLS by the following general equation

$$
n_{\eta}(v, \eta)=\vec{u} \cdot \nabla \Phi=\beta\left[v n_{\mu}+n_{\lambda}\right],
$$

where $\nabla=\left(\partial_{\mu}, \partial_{\lambda}\right)$ is the gradient in the $(\mu, \lambda)$ plane, and the r.h.s of the above formula is evaluated for

$$
\begin{aligned}
& \mu=v \beta \eta \\
& \lambda=\beta \eta
\end{aligned}
$$

Such a relation can be directly verified for the diffusion equation from Eqs. (8) of Ref. [2] and Eq. (29). Further, more significative tests have been performed by checking numerically the validity of Eq. (30) in some lattice models involving, e.g., logistic and homogeneous chains, the spectra of which are reported in see Fig. 5 (notice that, since the time units have not been renormalized in the tilted frame, the factor $\beta$ is no longer needed). 


\section{B. Entropy}

The connection between the chronotopic approach and the Kolmogorov-Sinai entropy $H_{K S}$ was already discussed in [2]. Here we recall the main concepts both for the sake of completeness and since it can be more effectively phrased with the help of the spatiotemporal representation.

As usual, we shall refer to Pesin formula [3] as a way to estimate the rate of informationproduction from the positive Lyapunov exponents. Although it provides an upper bound, there is numerical evidence that the bound is actually saturated in generic models. The reference to Pesin's formula suggests that $H_{K S}$ is an extensive quantity [6]. For this reason, it is convenient to introduce the entropy density $h_{\lambda}$ which, in the thermodynamic limit, can be obtained from the integral of the positive part of the Lyapunov spectrum.

It is natural to extend the definition of entropy to a generic reference frame,

$$
h_{\eta}=\int_{0}^{\eta_{\max }} n_{\eta}(\vartheta, \eta) d \eta
$$

where the integral is performed along the line $\mathcal{L}$ and $\eta_{\max }$ is the maximum value of $\eta$ which is reached in the intersection point between $\mathcal{L}$ and $\mathcal{D}$. In the limit $\vartheta=0$, the above equation reduces to the usual definition of $h_{\lambda}$, while for $\vartheta=\pi / 2$ it reduces to the "spatial" entropy $h_{\mu}$.

The existence of an entropy potential implies that

$$
h_{\eta}(\vartheta)= \begin{cases}h_{\lambda} & |\vartheta|<\vartheta_{*} \\ h_{\mu} & |\vartheta| \geq \vartheta_{*}\end{cases}
$$

where $\vartheta_{*}$ is the value for which the line $\mathcal{L}$ is tangent to $\mathcal{D}$. This is immediately seen by combining the observation that $h_{\eta}$ follows from an integral along the straight line defining the corresponding $\lambda$-axis with the observation that one of the two extrema is always the same (the origin) while the others lie along an equipotential line.

The independency of $h_{\eta}$ of $v$ has an immediate physical interpretation, which we also consider as a strong argument in favour of the existence of an entropy potential. The Kolmogorov-Sinai entropy density is, in fact, the amount of information necessary to characterize a space-time pattern of temporal duration $T$ and spatial extention $S$ (apart from the information flow through the boundaries [6]) divided by its area $S \times T$. Therefore, $h_{K S}$ is expected to be independent of the way the axes are oriented in the plane, i.e. of the velocity $v$. As a consequence, $h_{\eta}=h_{K S}$ for all $v<1$.

The above conclusion still holds when the STLS exhibits a positive band as well (which is always the case in continuous models), provided that the content of such a band is discarded. Accordingly, we can conclude that the new degrees of freedom, associated to the positive band, which appear in the rotated frame are just physically irrelevant directions which turn the original attractor into a repellor. If $v>c^{2} / V_{*}$, the two bands merge together and it is not anymore possible to distinguish between unstable but irrelevant directions and the unstable manifold of the original attractor. Presumably, this means that the repellor is turned into a strange repellor with a singular measure along some (all) unstable directions. 


\section{Dimension}

A second important indicator of the "complexity" of a spatiotemporal dynamics is the fractal dimension of the underlying measure. An upper bound $D_{K Y}$ to it is given by the Kaplan-Yorke formula [3]. The existence of a limit Lyapunov spectrum, implies that $D_{K Y}$ is proportional to the system size, so that it is convenient to introduce the dimension density $d_{K Y}$ [6], i.e. the number of independent degrees of freedom actively involved in the asymptotic evolution per unit length. In the framework of the present paper, it is natural to extend the concept of dimension density to a frame oriented in a generic way in the space-time plane. A straightforward generalization of the Kaplan-Yorke formula leads to the integral equation,

$$
\int_{0}^{d_{K Y}} \eta\left(v, n_{\eta}\right) d n_{\eta}=0
$$

As for the entropy density, Eq. (35) can be more easily interpreted with reference to the $\left(n_{\mu}, n_{\lambda}\right)$ plane. In this representation the entropy potential is

$$
\tilde{\Phi}=\lambda n_{\lambda}+\mu n_{\mu}-\Phi
$$

In fact, the curve implicitely defined by the constraint (35) is the equipotential line $\mathcal{C}$

$$
\tilde{\Phi}\left(n_{\mu}, n_{\lambda}\right)=0
$$

The dimension density $d_{K Y}(v)$ can, in turn, be determined from Eq. (30) at the intersection point between $\mathcal{C}$ and the image of $\mathcal{L}$ in the plane $\left(n_{\mu}, n_{\lambda}\right)$.

At variance with the entropy density, $d_{K Y}(v)$ changes with $v$ (see Fig. 6) even if we avoid considering the second positive band. In fact, while $h_{\eta}$ is an information divided by a space-time area, $d_{K Y}(v)$ is a number of degrees of freedom divided by a length, measured orthogonally to the propagation axis. Thus, at least from a dimensional point of view, it is meaningless to compare $d_{K Y}(v)$ for different velocities. However, one can reduce temporal to spatial lengths by introducing the scaling factor $c$ and, in turn, ask himself how the dimension changes with $c$. It is easily seen that the scaling dependence on $c$ is expressed by the following relation,

$$
d_{K Y}\left(v, c_{1}\right) \sqrt{1+\left(\frac{v}{c_{1}}\right)^{2}}=d_{K Y}\left(v, c_{2}\right) \sqrt{1+\left(\frac{v}{c_{2}}\right)^{2}}
$$

The (completely arbitrary) choice of $c$ reflects in different dependences of $d_{K Y}$ on $v$. A natural procedure to fix $c$ is by minimizing the dependence of $d_{K Y}$ on the observation angle. This amounts to choosing the time units in such a way as to make the 2D pattern as isotropic as possible. In homogeneous CMLs, the procedure is so effective that a suitable choice of $c$ allows removing almost completely the velocity dependence as seen in Fig. 6a, where the results for the natural value $c=1$ are compared with those for $c=3$.

More in general, however, it is not possible to achieve such a complete success. This is, for instance, the case of the logistic CML, where the dimension drop for $c=1$ is too large to be compensated by any choice of $c$ (see Fig. 6b, where the curve for $c=1$ is compared with the best results obtained for $c=+\infty)$. 
A further indicator which is sometimes useful in characterizing the chaoticity of a given extended system is the dimension density $d_{u}$ of the unstable manifold. This dimension is nothing but $n_{\eta}$ in the point where $\eta=0$, i.e. in the origin, and its expression simply reads as

$$
d_{u}=\beta n_{\lambda}(0,0) \quad .
$$

being $n_{\mu}(0,0) \equiv 0$. The choice $c=+\infty$ of the scaling factor removes exactly the dependence on the orientation of the reference frame. This choice is equivalent to measuring lengths in the untilted frame.

\section{CONCLUSIONS AND OPEN PROBLEMS}

In this paper we have provided further numerical evidence for the existence of an entropy potential in 1D spatially extended dynamical systems. The result appears to follow from the possibility to order the Lyapunov vectors according to their average wavenumber that thus becomes equivalent to the integrated density of exponents. This interpretation, if confirmed, would imply the possibility to define and attribute some meaning to the imaginary part of the expansion rates. This line of thought is very reminiscent of the rotation numbers introduced by Ruelle in the context of Hamiltonian systems [14]. It is certainly worth to fully explore this route in the hope to arrive at a more rigorous justification of our theoretical construction : work in this direction is in progress.

Furthermore, we have seen that, among the consequences of the existence of an entropy potential, there is the possibility to insert very coherently in this scheme a more general class of Lyapunov exponents corresponding to various directions of propagation in the space-time. As a result, we have found that the asymmetry between the temporal and the spatial axes can be revealed by the dependence of the density of dimension on the orientation of the reference frame. This is not true for the Kolmogorov-Sinai entropy density which, as already remarked in [2], should be regarded as a "super-invariant" dynamical indicator.

\section{ACKNOWLEDGMENTS}

We thank P. Grassberger, H. Kantz, and A. Pikovsky for useful discussions. We also acknowledge the hospitality of ISI-Torino during the activity of the EC Network CHRXCT94-0546, and one of us (A.T.) also the european contract ERBCHRX-CT94-0460. 


\section{REFERENCES}

[1] S. Lepri, A.Politi and A. Torcini, J. Stat. Phys. 821429 (1996).

[2] S. Lepri, A.Politi and A. Torcini, J. Stat. Phys. to appear (1997).

[3] D. Ruelle, J.P. Eckmann, Rev. Mod. Phys. 57, 617 (1985).

[4] Ya.G. Sinai Lectures in Ergodic Theory, Princeton U. Press, Princeton (1977).

[5] M.C. Cross and P.H. Hohenberg, Rev. Mod. Phys. 65, 851 (1993).

[6] P. Grassberger, Physica Scripta 40 (1989) 346.

[7] G. Giacomelli, S. Lepri and A. Politi, Phys. Rev. E 513939 (1995).

[8] I.S. Aranson, A.V. Gaponov-Greckhov, M.I. Rabinovitch and I.P. Starobinets, Sov. Phys. JETP 631000 (1986).

[9] K. Kaneko (ed.) Theory and Application of Coupled Map Lattices, John Wiley \& Sons (1993).

[10] R.J. Deissler and K. Kaneko, Phys. Lett. A 119397 (1987).

[11] I. Shimada and T. Nagashima, Prog. Theor. Phys. 61, 1605 (1979); G. Benettin, L. Galgani, A. Giorgilli and J.M. Strelcyn, Meccanica, 1521 (1980).

[12] A.Torcini, P. Grassberger and A. Politi, J. Phys. A. 274533 (1995).

[13] A. Politi and A. Torcini, Europhys. Lett. 28545 (1994).

[14] D. Ruelle, Ann. Inst. Poincaré, 42, 109 (1985). 


\section{FIGURES}

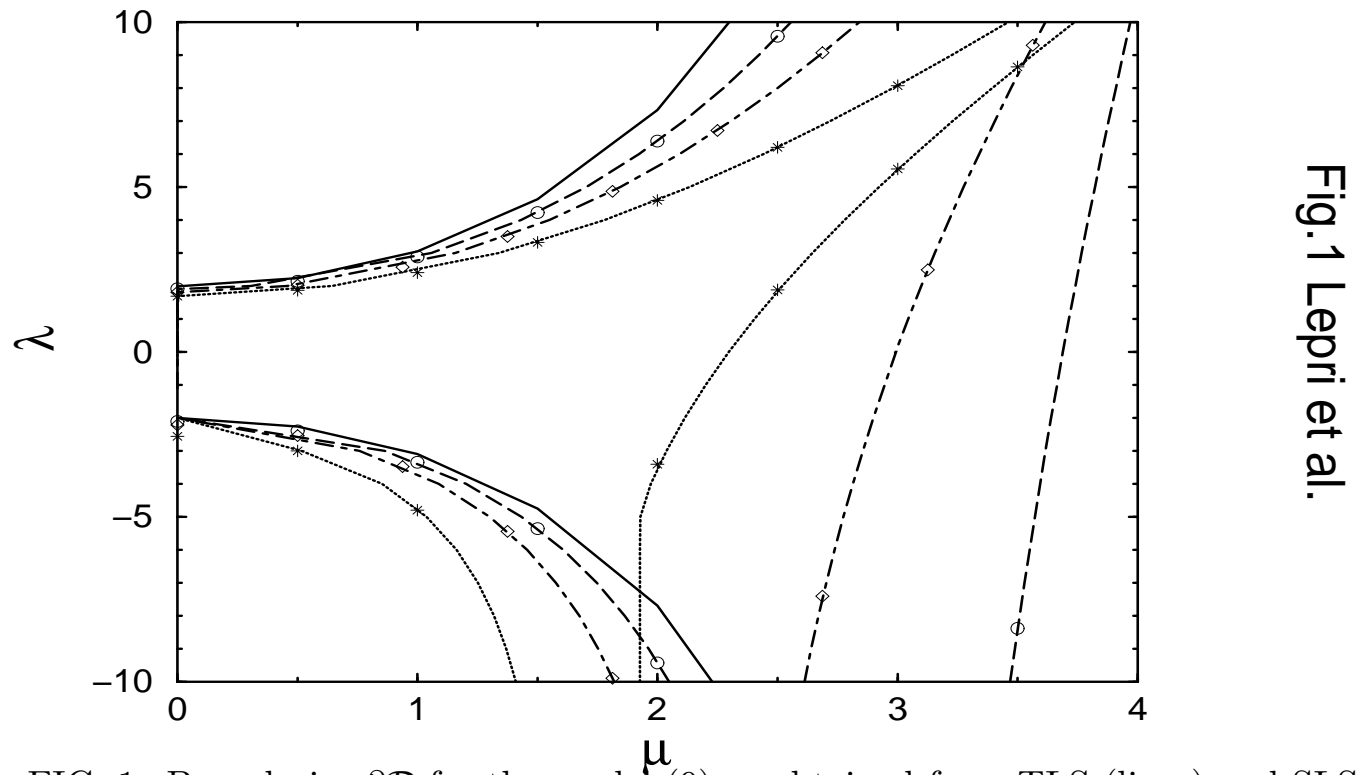

FIG. 1. Boundaries $\partial \mathcal{D}$ for the model (9) as obtained from TLS (lines) and SLS (symbols) for three different values of the time step $\Delta t: 0.2$ (dotted line and asterisks), 0.1 (dot-dashed line and diamonds) and 0.05 (dashed line and circles). The asymprotic boundaries are also reported for $\Delta t=0.00625$ (solid line).

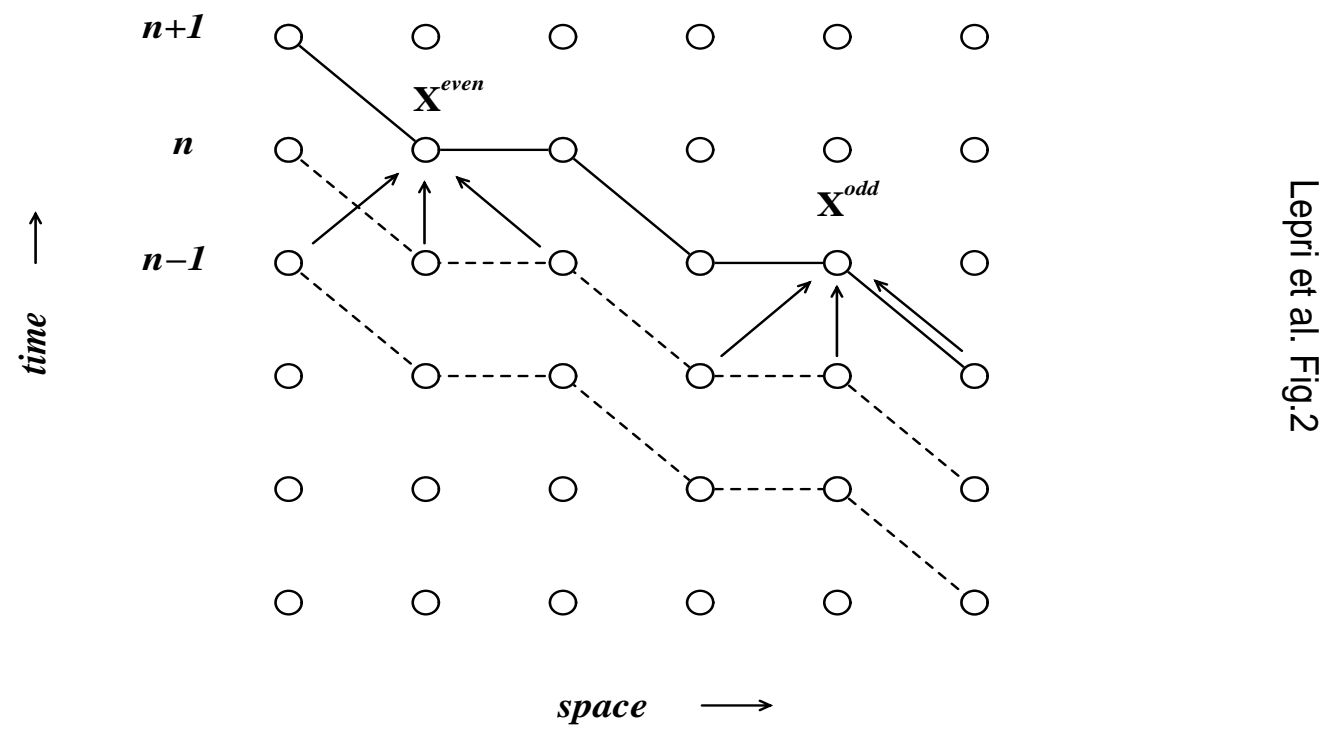

FIG. 2. Lattice implementation of the definition of spatiotemporal Lyapunov exponents for $v=1 / 2$. 


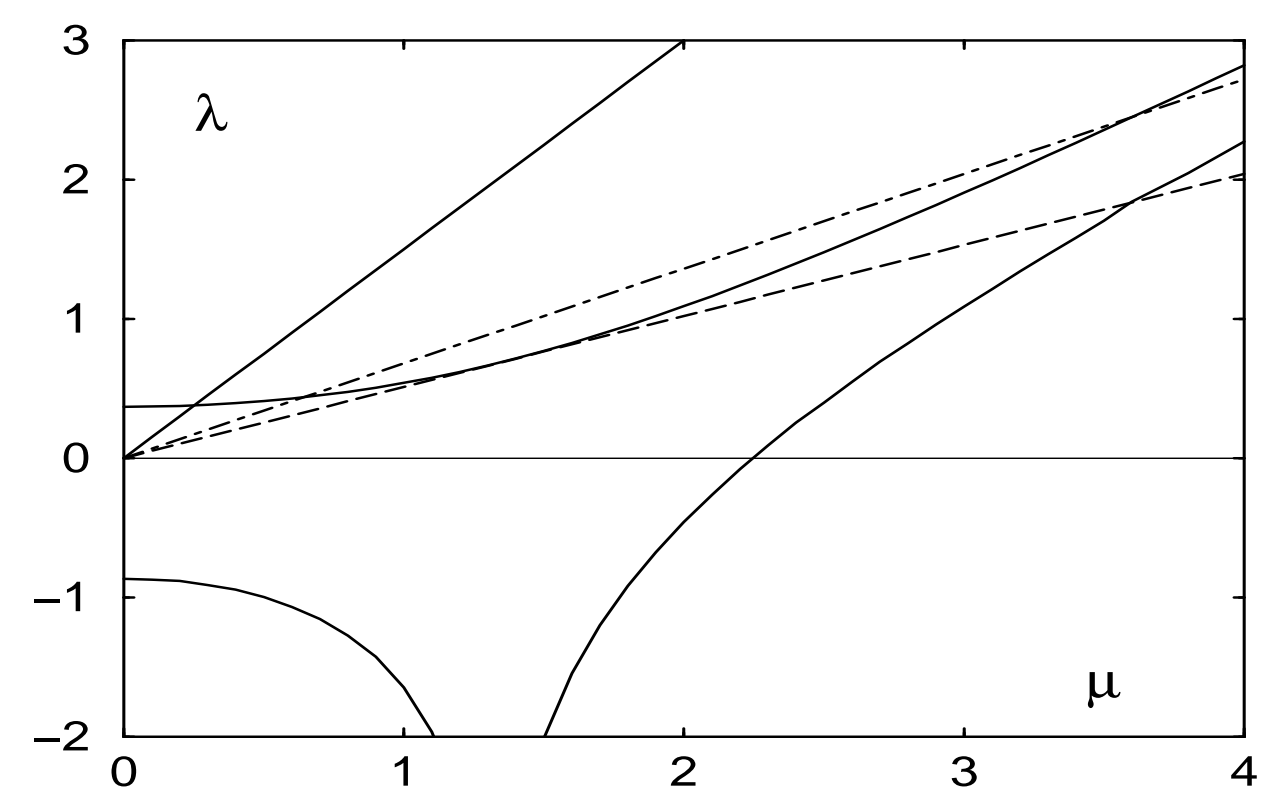

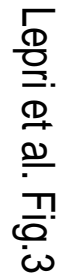

FIG. 3. Plot of the boundary $\partial \mathcal{D}$ and of the line $\lambda=v \mu$ in the three velocity regimes for the logistic CML $\varepsilon=1 / 3$. The three lines refer to the different cases $v<1$ (solid), $1<v<1 / V_{*}$ (dot-dashed) and $v=1 / V_{*}$ (dashed).

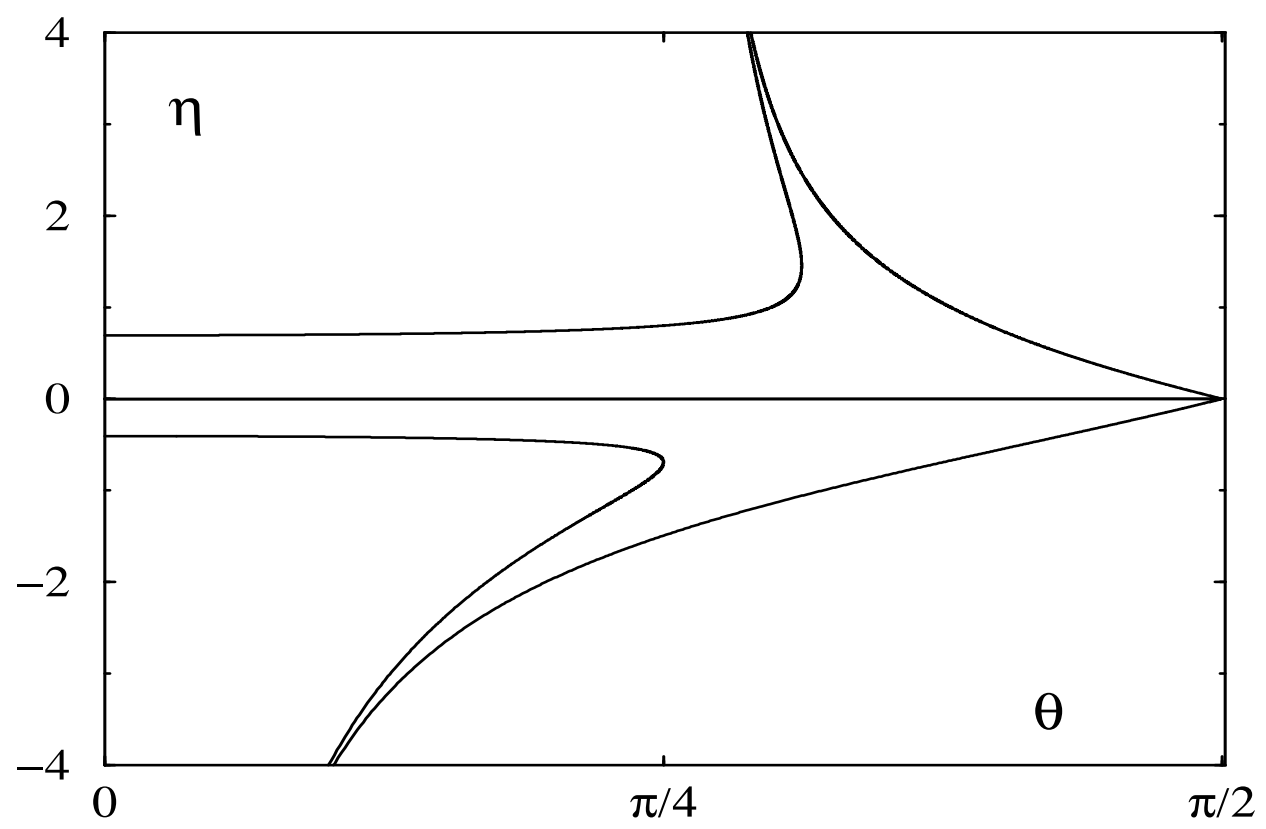

FIG. 4. Boundaries of the STLS versus the tilting angle $\vartheta$ for the homogeneous chain $(r=2$, $\varepsilon=1 / 3)$. 

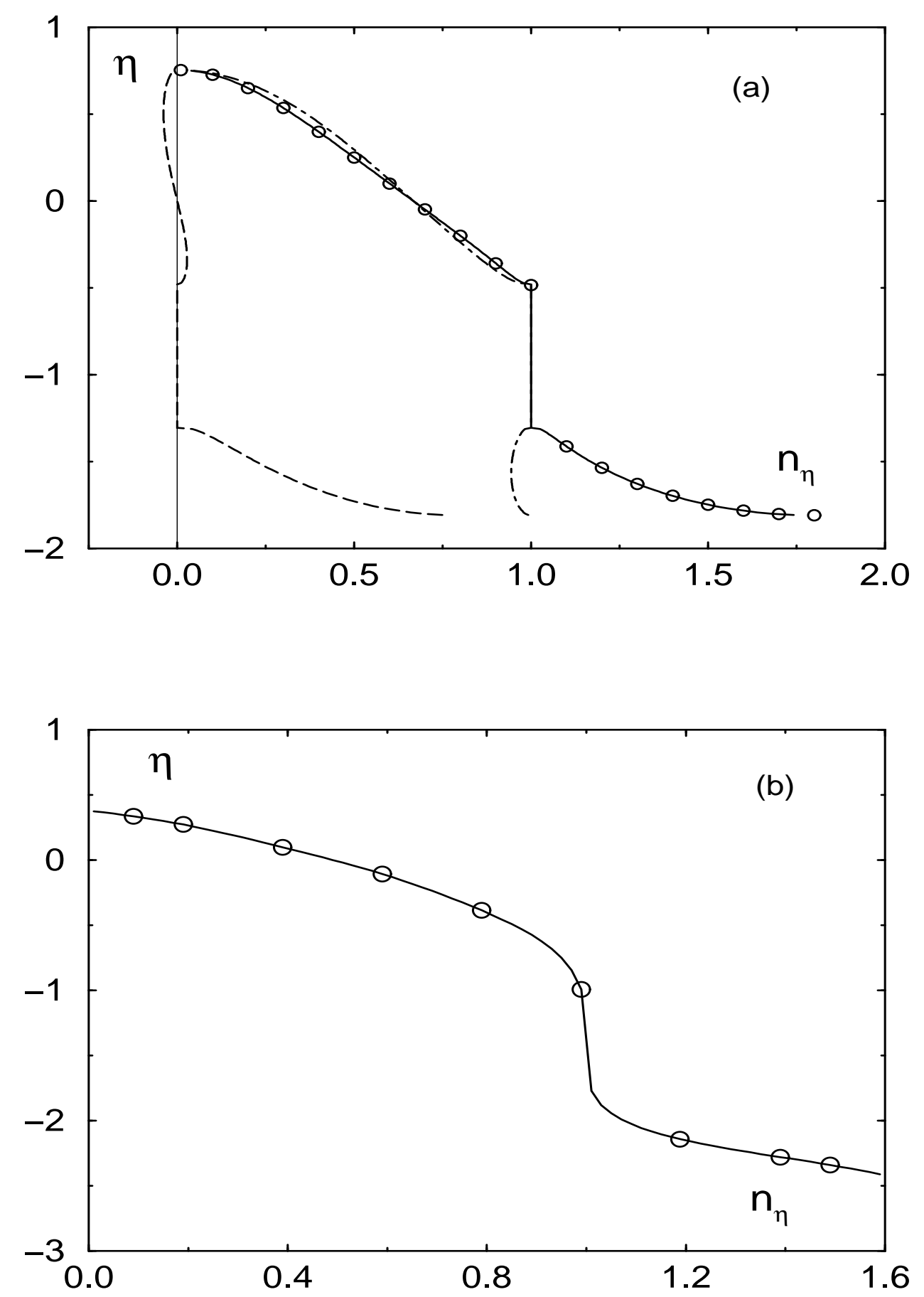

FIG. 5. Comparison between the STLS obtained by direct numerical computation and formula (30) for (a) homogeneous $(r=2)$ with $v=4 / 5$ and (b) logistic CMLs with $v=3 / 5$ (in both cases $\varepsilon=1 / 3)$. 

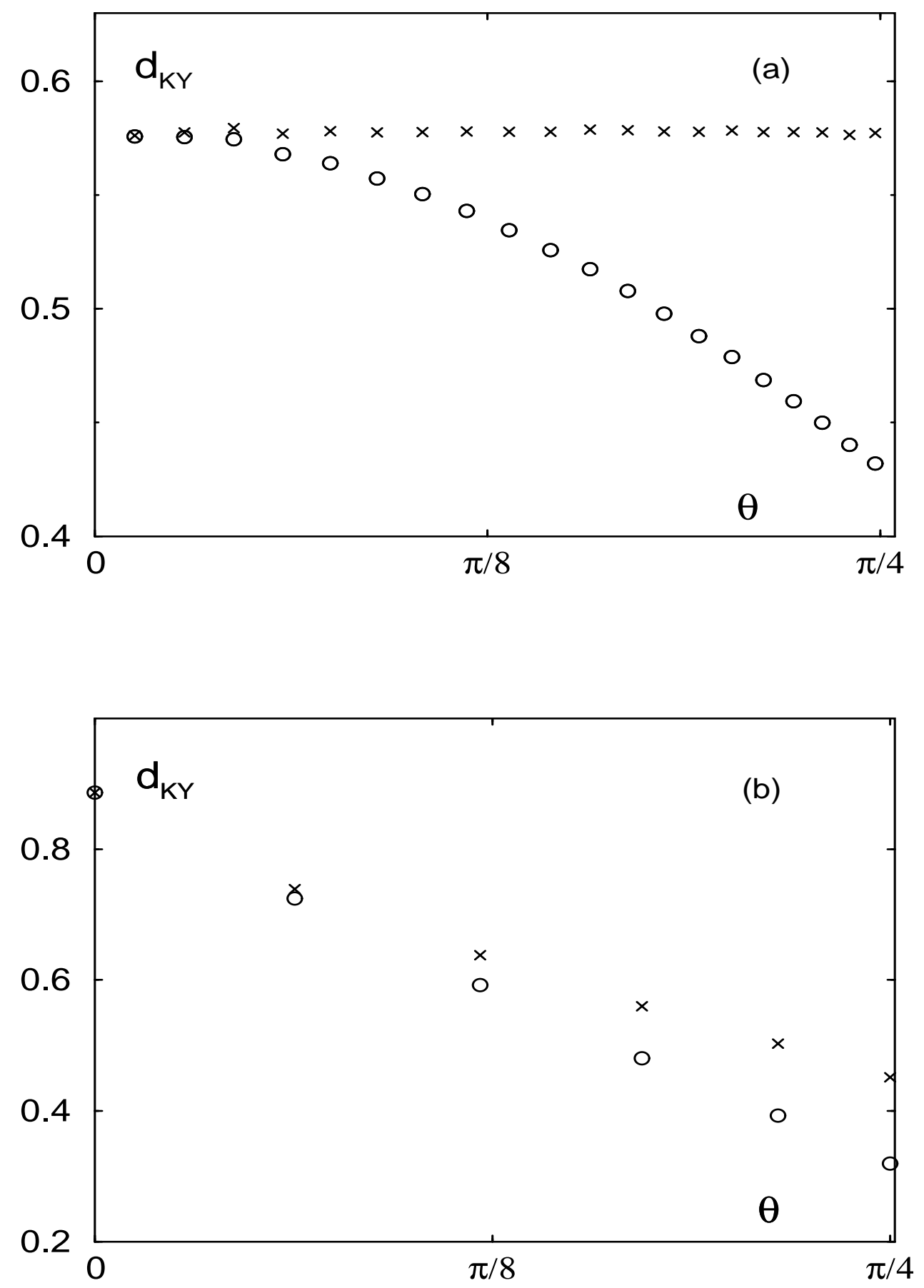

FIG. 6. Kaplan-Yorke dimension density $d_{K Y}$ obtained from the STLS versus the tilting angle $\vartheta$ for (a) homogeneous $(r=1.2)$ and (b) logistic CMLs: in both cases $\varepsilon=1 / 3$. Circles refer to the scaling factor $c=1$, while crosses correspond to $c=3,+\infty$ in (a), (b), respectively. 


\section{TABLES}

\begin{tabular}{cccc}
\hline \hline Path & $\Delta t=0.2$ & $\Delta t=0.1$ & $\Delta t=0.05$ \\
\hline \hline$(0,0) \rightarrow(0,1) \rightarrow(2,1)$ & -1.1419 & -1.1868 & -1.2303 \\
$(0,0) \rightarrow(2,0) \rightarrow(2,1)$ & -1.1358 & -1.1838 & -1.2236 \\
& & & \\
Relative difference & $0.5 \%$ & $0.2 \%$ & $0.5 \%$ \\
\hline \hline
\end{tabular}

TABLE I. Entropy potential $\Phi$ for model (9) $\left(S_{0}=1 / 3, \sigma=0.1, \omega_{0}=1, D=1\right)$ computed by integrating along two different piecewise linear paths of the $(\mu, \lambda)$ plane, and for three different time steps. In the last row the relative difference between the two values is reported: relative statistical error in the computation of Lyapunov exponents are of the order of $10^{-3}$. 